\title{
Role of DNA testing for diagnosis, management, and genetic screening in long QT syndrome, hypertrophic cardiomyopathy, and Marfan syndrome
}

In recent years the molecular genetics and pathophysiology of long QT syndrome (LQTS), hypertrophic cardiomyopathy (HCM), and Marfan syndrome have been extensively studied. Each disease shows highly variable expression and reduced penetrance of all known phenotypic manifestations ${ }^{1-3}$ and the diagnosis is often difficult. In addition, the possibility of genotype specific treatment has been raised for LQTS. As a consequence of these diagnostic and therapeutic issues, the role of DNA analysis (genotyping) has become a topic of substantial interest. ${ }^{4-6}$ Further, as each of these diseases causes unexpected sudden death in children and young adults, often during physical activity, the role of athletic preparticipation screening, ${ }^{7}$ including DNA testing, is now a widely discussed topic. This editorial will, therefore, examine the role of genotyping for: (1) diagnosis and management of individual patients; and (2) for "genetic screening", the identification of these diseases in populations such as young athletes.

\section{When is DNA analysis likely to be helpful for the diagnosis and management of patients?}

(1) When the diagnosis is difficult, often because of "reduced penetrance" and "variable expressivity". Reduced penetrance refers to the absence in some patients of the phenotypic features of the disease. Variable expressivity refers to a high degree of variability of the phenotype.

(2) When the clinical course varies importantly by genotype or mutation type, and the genetic information will alter treatment strategies. For example, the identification of genotypes predisposing to breast cancer has encouraged some genetically predisposed patients to have prophylactic mastectomy. Or, if one particular genotype in LQTS or HCM was found to be associated with a particularly high rate of ventricular fibrillation and sudden death, prophylactic insertion of an automatic implantable cardioverter-defibrillator (ICD) might be considered. However, if there is no compelling "need to know", current economic constraints and ethical/privacy issues may limit the interest of governments or insurance companies to pay for DNA testing.

When is genetic screening feasible?

(1) When the disease causing gene(s) and all mutations are known. Otherwise, patients with the disease, but with mutations of undiscovered genes, will be "false negatives". This would complicate, rather than clarify, the diagnosis and treatment.

(2) When the disease is caused by a mutation(s) of a single gene. When mutations of multiple genes are required, called "polygenic disorders", or when both genetic abnormalities and environmental factors are required in order for the disease to develop, molecular genetic studies may not be fully informative or as useful.

So how can these concepts can be applied to LQTS, HCM, and Marfan syndrome?

\section{Long QT syndrome}

Inherited LQTS ${ }^{1}$ is an autosomal dominant disorder (in Jervell, Lange-Nielsen syndrome the deafness is recessive, but the LQTS is dominant) with an estimated prevalence of 1:5000 persons. It is effectively treated with $\beta$ blockers, implantable defibrillators, and pacemakers. Five genes which encode cardiac ion channels and about 180 mutations have been reported. ${ }^{4}$ Sporadic cases occur but are uncommon. No mutation is found on genotyping in about $30 \%$ of clinically affected patients. Some of these patients may have mutations of undiscovered genes, and some cases may be due to limitations of the analysis techniques. There is tremendous variability of expression and reduced penetrance of the QTc and the symptom phenotypes. For example, fig 1 shows the QTc distribution on baseline ECG of a large number of LQTS gene carriers. Graphs of the characteristic phenotype of HCM and Marfan syndrome would show similar variability. Between a third and a half of gene carriers never have symptoms. Approximately $4-5 \%$ of gene carriers experience sudden death.

What about DNA analysis for diagnosis and management? Genotyping can be very important for the approximately 35\% (fig 1) of gene carriers with normal to borderline QTc intervals of $0.41-0.46$ seconds, since they are difficult to diagnose and to separate from the large percentage of normals with these same QTc values. Importantly, recent evidence indicates these gene carriers with reduced penetrance of the QTc have essentially the same risk of syncope and sudden death as LQTS patients as a whole. ${ }^{8}$ In them, a correct diagnosis allows life saving treatment to be instituted. This can be quite important in evaluating relatives of a known LQTS patient or an unexplained sudden death victim. While no commercial laboratories perform DNA tests for LQTS, a number of research labs in the USA, Europe, Japan, and elsewhere do such genotyping on a limited basis, and can be contacted by interested physicians. Genotype specific treatment has

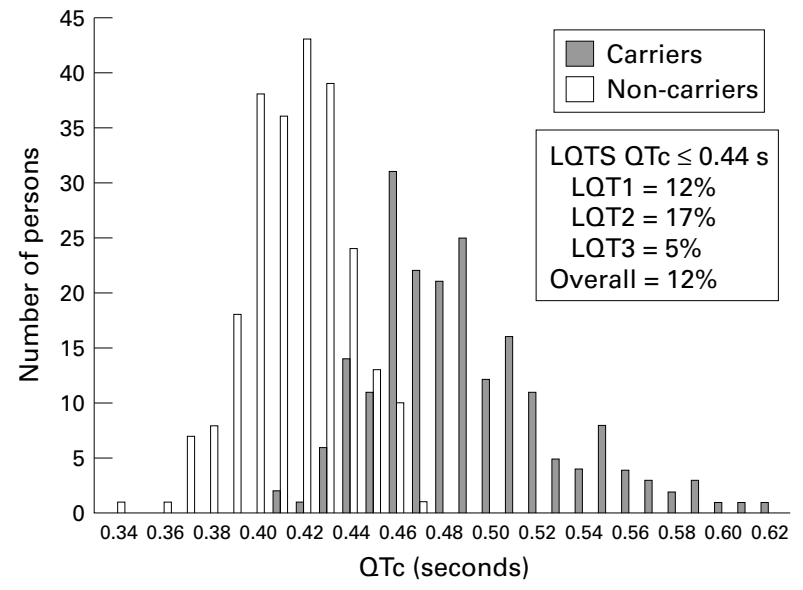

Figure 1 Range of QTc intervals on baseline ECG of 208 LQTS gene carriers (LQT1, LQT2, and LQT3) and 288 non-gene carriers. There is a large overlap between carriers and non-carriers. Reduced penetrance is evident: a normal interval (QTc $\leqslant 0.44$ seconds) was present in $12 \%$ of gene carriers, variable by genotype, and a normal to borderline prolonged interval (QTc $\leqslant 0.46$ seconds) was present in $36 \%$. 
been proposed in LQTS - for example, sodium blocker drugs rather than $\beta$ blockers for LQT3, and potassium therapy for LQT2. There are no data yet on the effectiveness of these regimens for prevention of syncope or sudden death, nor the risk of these treatments. Therefore, there is no current indication for the routine use of these treatments, and no need for genotyping for this purpose.

What about genetic screening of all children, young athletes, or all young persons with unexplained syncope? No commercial laboratories do these tests because of the cost and the false negatives as noted above. Further, the false positive test rate is not well defined, but would have to be extremely low for a screening test. Thus, population screening for LQTS by DNA testing is neither recommended nor available.

\section{Hypertrophic cardiomyopathy}

HCM is an autosomal dominant disease with a prevalence estimated at 1:500 persons. Occasional sporadic cases are identified. Nine different genes encoding for sarcomere proteins and more than 110 mutations have been identified. ${ }^{2}$ Similar to LQTS, no mutation is found in about $30 \%$ of clinically affected patients. Diagnosis is primarily based on echocardiographic demonstration of ventricular hypertrophy, but there is major variability of expression and reduced penetrance. The phenotype and prognosis vary tremendously within as well as between genotypes. A few families have been described with higher or lower than average incidences of sudden death. However, these families are relatively small and the difference in incidence of events might be random chance. More recent observations on larger pedigrees suggest this to be the case. Pharmacologic treatment is beneficial in reducing cardiac hypertrophy and heart failure signs and symptoms, but there is no clear evidence that treatment alters the risk of sudden death. No parameter predicts sudden death, so automatic ICD implantation is not suitable as a prophylactic intervention.

To complicate HCM genetic testing, some features such as valvulopathy are probably unrelated to the sarcomere gene mutations. ${ }^{6}$ This observation suggests that additional genetic and/or environmental factors play a role in the expression and presumably the outcome of the disease; thus, the results of sarcomere gene analyses alone may not clarify either the treatment options or the expected outcome. Because of these factors, no commercial laboratories perform DNA tests for HCM, but they are done in a number of research labs in the USA and elsewhere.

What about DNA analysis for diagnosis and management? Genetic testing could certainly improve diagnostic accuracy in the gene carriers with reduced penetrance of ventricular hypertrophy. As noted above, however, knowing the diagnosis, genotype or mutation type does not clearly influence treatment options or outcome. Thus, there seems to be no compelling reason to perform genotyping for these clinical purposes. ${ }^{9}$ If effective treatment strategies are identified, genotyping would become of great importance, as in LQTS patients with reduced penetrance.

What about genetic screening of young athletes? The sudden death of a young athlete who is found to have $\mathrm{HCM}$ at necropsy is often cited as a reason to screen for this disease. However, the cost, false negatives, possible false positives, and lack of clear usefulness of the genetic result preclude the use of DNA analyses for general genetic screening at the present time. ${ }^{9}$

\section{Marfan syndrome}

Marfan syndrome ${ }^{3}$ is an autosomal dominant disorder with an estimated prevalence of 1:5000 persons. It is caused by mutations in the FBN1 gene on chromosome 15q, which encodes for the connective tissue protein fibrillin-1.
Approximately 125 mutations of this large gene have been identified, and nearly every family has its own novel mutation. About $75 \%$ of cases are inherited, and $25 \%$ are sporadic caused by de novo mutations. The typical phenotype includes the well known skeletal, ocular, and cardiovascular manifestations. Aortic aneurysm, dissection, and rupture are the most serious and the cause of sudden death. As with LQTS and HCM, there is tremendous variability of expression of the phenotype, and reduced penetrance. Similar to HCM, Marfan syndrome exhibits a number of anatomic abnormalities, which are not clearly related to the FBN1 mutations. ${ }^{6}$ Their role in the outcome of the disease is unclear. Importantly, FBN1 gene mutations are sometimes found in the absence of any of the diagnostic criteria for Marfan syndrome. Apparently the mutations are not specific for Marfan syndrome, though these individuals might have the syndrome with reduced penetrance of all phenotypic features. Currently, such subjects are not given the diagnosis of Marfan syndrome.

Treatment with medications, lifestyle changes, and prophylactic aortic surgery does influence the outcome of this disease. However, treatment is instituted based on echocardiographic findings of the aorta, not the genetic findings. No commercial laboratories perform genotyping for Marfan syndrome.

What about DNA analysis for diagnosis and management? At present, there is not a good rationale for routine genotyping of suspected or known Marfan syndrome patients. Genetic information does not alter the treatment strategy, and does not clarify the diagnosis in patients without the typical phenotype, because of the false positive results.

What about genetic screening? With no apparent advantage for diagnosis or patient care, there is no reason to perform screening examinations.

\section{Conclusion}

Genotyping can be very important for diagnosis in LQTS, but not, at present, for HCM or Marfan syndrome. Routine management is not genotype dependent in any of the diseases. Genetic screening certainly has the potential to improve medical care greatly in genetically mediated diseases. However, it is not suitable for LQTS, HCM or Marfan syndrome at present for the reasons stated above. Before widespread screening for these and other disorders is undertaken several advances will be necessary. All genes and mutations must be identified. Which populations could benefit from DNA screening must be determined and the cost:benefit ratio must be acceptable to both payors and patients. A rapid, reasonably priced, and accurate analysis technique for multiple genes and mutations will be required. The DNA chip and other emerging technologies will likely provide this advance. Lastly, society and the legal and medical professions must agree on, and develop appropriate guidelines for, the ethical management of genetic data in order to protect patients from the inappropriate use and exploitation of these data, both within and outside the health care profession.

G MICHAEL VINCENT

Department of Medicine,

LDS Hospital and University of Utah,

Salt Lake City, UT 84143, USA

ldgvince@ihc.com

1 Vincent GM. Long QT syndrome. Cardiol Clin 2000;18:309-25.

2 Burch M, Blair E. The inheritance of hypertrophic cardiomyopathy. Pediatr Cardiol 1999;20:313-16.

3 Pyeritz RE. The Marfan syndrome. Ann Rev Med 2000;51:481-510.

4 Splawski I, Shen J, Timothy KW, et al. Spectrum of mutations in long-QT syndrome genes KVLQT1, HERG, SCN5A, KCNE1, and KCNE2. Circu-
sydrions in longlation 2000;102:1178-85. 
5 Burn J, Camm J, Davies MJ, et al. The phenotype/genotype relation and the current status of genetic screening in hypertrophic cardiomyopathy, Marfan

6 Maron BJ, Moller JH, Seidman CE, et al. Impact of laboratory molecular diagnosis on contemporary diagnostic criteria for genetically transmitted cardiovascular diseases: hypertrophic cardiomyopathy, long QT syndrome and marfan syndrome. Circulation 1998;98:1960-71.
7 Pfister GC, Puffer JC, Maron BJ. Preparticipation cardiovascular screening Pfister GC, Puffer JC, Maron BJ. Preparticipation cardiovas Vincent GM, Zhang L, Timothy KW, et al. Long QT syndrome patients with normal to borderline prolonged QTc intervals are at risk for syncope, cardiac arrest and sudden death [abstract]. Circulation 1999;100:1272.

9 Thierfelder L. Genetic screening of cardiomyopathies [German, English abstract]. Zeitschrift fur Kardiologie 2000;89:638-40.

\section{Electrical interference between two transvenous implantable defibrillator leads}

A 52 year old male patient with an implantable cardioverter/defibrillator (ICD) and a transvenous ICD lead (Endotac 0062, Guidant Inc, St Paul, Minnesota, USA), who presented with electrical lead desintegrity with inappropriate shock delivery, received a new transvenous ICD lead (Sprint 6942, Medtronic Inc, Minneapolis, Minnesota, USA) without extraction of the old lead. The implantation of the new ICD lead was complicated by difficulties in finding a position with satisfactory sensing and pacing properties and adequate defibrillation efficacy. The final lead position was in very close proximity to the distal coil of the former implanted lead (below left). During device based intraoperative testing high frequency noise signals were observed in the rate sensing channel after shock delivery of defibrillation energies from $31 \mathrm{~J}$ down to $6 \mathrm{~J}$ (below right, arrows). The sensing of these artefacts led to inappropriate redetection of ventricular fibrillation with consecutive shock delivery.
By reducing the maximum sensitivity level of the device (MINI IV, Guidant Inc) from $0.14 \mathrm{mV}$ to $0.28 \mathrm{mV}$, sensing of induced ventricular fibrillation was not influenced but noise detection after shock delivery could be completely prevented.

Since 199012 patients underwent implantation of a second ICD lead without extraction of the old lead in our hospital. This is the first case of electrical interference of the two leads, which can be explained by their very close proximity.

Thus, if the implantation of a second ICD lead is necessary, positioning the leads too close to one another should be avoided. Post-shock intracardiac electrograms should be analysed carefully to exclude electrical interference between the two implanted leads.
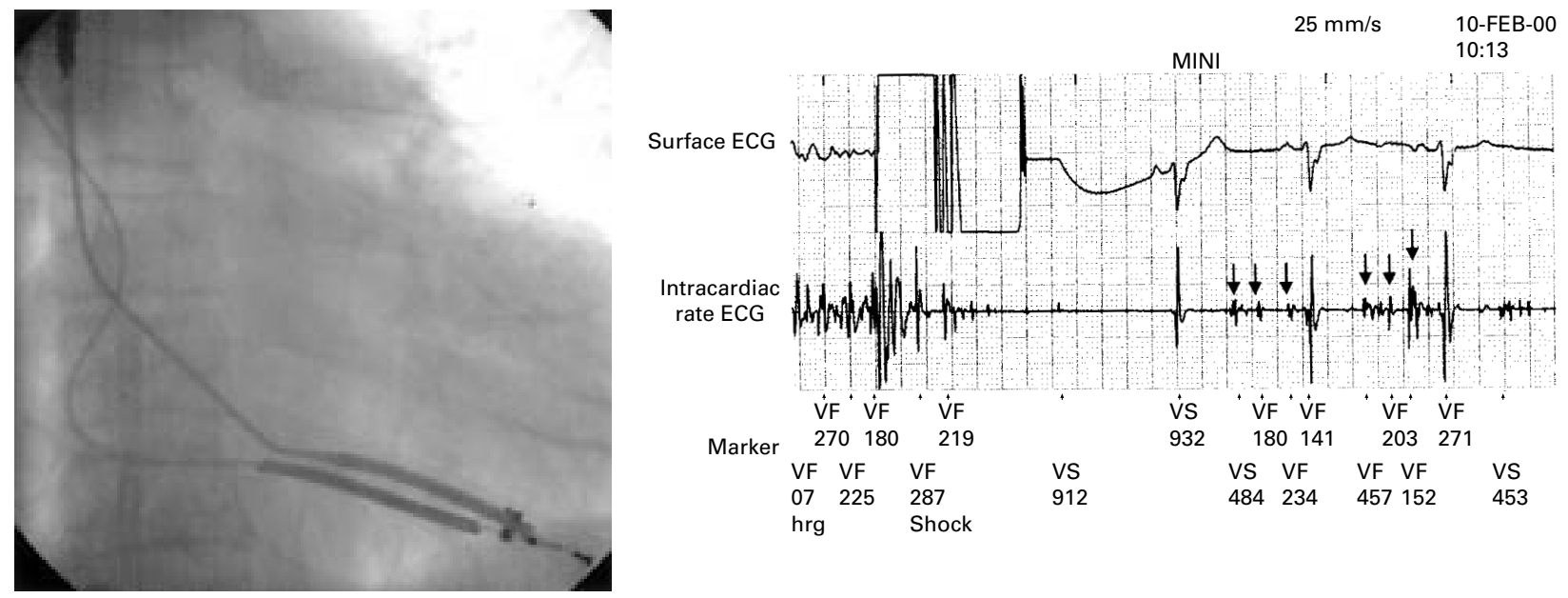